Pacific Journal of Mathematics

ON THE BARYCENTRIC HOMOMORPHISM IN A SINGULAR

COMpLEX 


\title{
ON THE BARYCENTRIC HOMOMORPHISM IN A SINGULAR COMPLEX
}

\author{
PaUl V. ReICHELderfer
}

\section{INTR ODUCTION}

0.1. Radó has introduced and studied the following approach to singular homology theory (see $[2 ; 3 ; 4]$ for details). With a general topological space $X$ associate a complex $R=R(X)$ in the following manner. For integers $p \geq 0$, let $v_{0}, \cdots, v_{p}$ be a sequence of $p+1$ points in Hilbert space $E_{\infty}$, which are not required to be distinct or linearly independent, and let $\left|v_{0}, \cdots, v_{p}\right|$ denote their convex hull. Suppose that $T$ is a continuous mapping from $\left|v_{0}, \cdots, v_{p}\right|$ into $X$. Then the sequence $v_{0}, \cdots, v_{p}$ jointly with $T$ determines a $p$-cell in $R$, which is denoted by $\left(v_{0}, \cdots, v_{p}, T\right)^{R}$. The free Abelian group $C_{p}^{R}$ generated by the $p$-cells in $R$ is termed the group of integral $p$-chains in $R$. For integers $p<0, C_{p}^{R}$ is defined to be the group consisting of the zero element alone. The boundary operator $\partial_{p}^{R}: C_{p}^{R} \rightarrow C_{p-1}^{R}$ is defined, in the usual manner, as the trivial homomorphism if $p \leq 0$, and by the relation

$$
\partial_{p}^{R}\left(v_{0}, \cdots, v_{p}, T\right)^{R}=\sum_{i=0}^{p}(-1)^{p}\left(v_{0}, \cdots, \hat{v}_{i}, \cdots, v_{p}, T\right)^{R}
$$

if $p>0$. Since $\partial_{p-1}^{R} \partial_{p}^{R}=0$, one introduces the subgroup $Z_{p}^{R}$ of $p$-cycles in $C_{p}^{R}$ and the subgroup $B_{p}^{R}$ of $p$-boundaries in $C_{p}^{R}$ in the customary way, and defines the quotient group of $Z_{p}^{R}$ with respect to $B_{p}^{R}$ to be the homology group $H_{p}^{R}$.

0.2. The approach to singular homology theory pursued by Radó differs from other approaches in that absolutely no identifications are made. Thus two $p$-cells $\left(v_{0}^{\prime}, \cdots, v_{p}^{\prime}, T^{\prime}\right)^{R}$ and $\left(v_{0}^{\prime \prime}, \cdots, v_{p}^{\prime \prime}, T^{\prime \prime}\right)^{R}$ are equal only if they are identical; that is, if $v_{i}^{\prime}=v_{i}^{\prime \prime}$ for $i=0, \cdots, p$ and $T^{\prime} \equiv T^{\prime \prime}$ on $\left|v_{0}^{\prime}, \ldots, v_{p}^{\prime}\right|$ $=\left|v_{0}^{\prime \prime}, \cdots, v_{p}^{\prime \prime}\right|$. In [3;4], Radó introduces a technique for making identifications in a general Mayer complex and applies his procedure to study identifications in $R$, particularly those which yield homology groups isomorphic to the $H_{p}^{R}$. It is a primary purpose of the present paper to pursue the matter further in

Received January 24, 1951.

Pacific J. Math. 2 (1952), 73-97 
order to establish stronger results than those obtained by Radó.

The identification scheme of Radó for the complex $R$ is briefly described in $\S 0.3$ below; the reader should consult $[3, \S 1]$ or $[4, \S 5]$ for details.

0.3. Let $\left\{G_{p}\right\}$ be a collection of subgroups $G_{p}$ of the group $C_{p}^{R}$ of integral $p$-chains in $R$ such that $\partial_{p}^{R} G_{p} \subset G_{p-1}$ for every integer $p$; such a system is termed an identifier for $R$. Let $C_{p}^{m}$ be the quotient group of $C_{p}^{R}$ with respect to $G_{p}$, and denote that element of $C_{p}^{m}$ to which a chain $c_{p}^{R}$ in $C_{p}^{R}$ belongs by $\left\{c_{p}^{R}\right\}_{\text {. }}$ The restriction on the groups $G_{p}$ clearly implies that the element $\left\{\partial_{p}^{R} c_{p}^{R}\right\}$ in $C_{p-1}^{m}$ is independent of the choice of the representative $c_{p}^{R}$ of the element $\left\{c_{p}^{R}\right\}$ in $C_{p}^{m}$; thus one may define homomorphisms $\partial_{p}^{m}: C_{p}^{m} \rightarrow C_{p-1}^{m}$ by the formula $\partial_{p}^{m}\left\{c_{p}^{R}\right\}=\left\{\partial_{p}^{R} c_{p}^{R}\right\}$. The resulting system of groups $C_{p}^{m}$ together with the operator $\partial_{p}^{m}$ constitutes a Mayer complex $m$ with homology groups $H_{p}^{m}$. Define a natural homomorphism $\pi_{p}: C_{p}^{R} \rightarrow C_{p}^{m}$ by the formula $\pi_{p} c_{p}^{R}=\left\{c_{p}^{R}\right\}$. It is readily verified that $\pi_{p}$ is a chain mapping; hence it induces homomorphisms $\pi_{*}: H_{p}^{R} \rightarrow H_{p}^{m}$. If for every integer $p$ these homomorphisms are isomorphisms onto, then the identifier $\left\{G_{p}\right\}$ is termed unessential for $R$. Radó notes that a necessary and sufficient condition in order that an identifier $G_{p}$ be unessential for $R$ is that every cycle $z_{p}^{R}$ in $G_{p}$ should be the boundary of some chain $c_{p+1}^{R}$ in $G_{p+1}$. (See [3, $\S \S 1.3,1.4,1.5]$ or $[4, \S 5]$.)

0.4. One of the principal results in this paper may now be described. Let $\beta_{p}^{R}: C_{p}^{R} \rightarrow C_{p}^{R}$ be the barycentric homomorphism in $R$ (see $[3, \S 3.1]$ or $[4, \S 6]$; also $\$ 1.3)$, and denote by $N\left(\beta_{p}^{R}\right)$ the nucleus of this homomorphism for every integer $p$.

THEOREM. The system of nuclei $N\left(\beta_{p}^{R}\right)$ of the barycentric homomorphisms in in $R$ constitutes an unessential identifier for $R$ (see $\$ 3.2$ ).

This result is combined with those of Radó in [3] to obtain stronger theorems concerning identifiers than any previously obtained. Since further definitions are necessary before these results can be described, the reader is requested to consult $\S 3$ for the ir statements.

0.5. In the process of proving the the orem above, various results of independent interest have been attained. The reader is referred especially to $\$ \$ 1.6$, $1.7,1.10,2.2$ for theorems which show the structural description of the barycentric homomorphism and of the barycentric homotopy operator. 


\section{Further Relations in the Auxiliary Complex $K$}

1.1. As in Rado [3;4], the auxiliary complex $K$ is the "formal complex", in the sense of [1], for the set $E_{\infty}$ of points in Hilbert space. For integers $p \geq 0$, $p$-cells in $K$ are ordered sequences $\left(v_{0}, \ldots, v_{p}\right)$ of $p+1$ points in $E_{\infty}$, which are not required to be distinct or linearly independent. These $p$-cells are taken as the base for a free Abelian group $C_{p}$, which is termed the group of finite integral $p$-chains in $K$. For $p<0$, the group $C_{p}$ is defined to be the group composed of the zero element alone. (See $[3, \S 2.1]$ or $[4, \S 6]$.) $[4, \$ 6]$.

1.2. In $K$ the following known homomorphisms will be used. (See [3, \$2.2] or

(i) For integers $j, p$ such that $0 \leq j \leq p, p>0$, the homomorphism

$$
j_{p}: C_{p} \rightarrow C_{p-1}
$$

is defined by the relation $j_{p}\left(v_{0}, \cdots, v_{p}\right)=(-1)^{j}\left(v_{0}, \ldots, \hat{v}_{j}, \ldots, v_{p}\right)$, where the symbol ${ }^{\wedge}$ is placed over the point $v_{j}$ to indicate that $v_{j}$ is to be deleted. For $j=p=0, j_{p}$ is defined to be the trivial homomorphism. A homomorphism differing from this one only by the absence of the factor $(-1)^{j}$ has been used by Rado in $[2, \S 2.6]$. The definition given above has been chosen because it permits simplifications in later definitions and formulas.

(ii) For integers $p>0$, the boundary operator

$$
\partial_{p}: C_{p} \rightarrow C_{p-1}
$$

is defined by the formula

$$
\partial_{p}\left(v_{0}, \cdots, v_{p}\right)=\sum_{j=0}^{p}(-1)^{j}\left(v_{0}, \cdots, \hat{v}_{j}, \cdots, v_{p}\right) .
$$

For integers $p \leq 0, \partial_{p}$ is defined to be the trivial homomorphism.

(iii) For integers $p \geq 0$ and an arbitrary point $v$ in $E_{\infty}$, the cone homomorphism $h_{p}^{v}: C_{p} \longrightarrow C_{p+1}$ is defined by the relation

$$
h_{p}^{v}\left(v_{0}, \cdots, v_{p}\right)=(-1)^{p+1}\left(v_{0}, \ldots, v_{p}, v\right) .
$$

For integers $p<0, h_{p}^{v}$ is defined to be the trivial homomorphism.

(iv) For integers $j, p$ such that $0 \leq j \leq p-1$, the transposition homomorphism $t_{p j}: C_{p} \rightarrow C_{p}$ is defined by the relation 


$$
t_{p j}\left(v_{0}, \cdots, v_{j}, v_{j+1}, \cdots, v_{p}\right)=\left(v_{0}, \ldots, v_{j+1}, v_{j}, \cdots, v_{p}\right) .
$$

Observe that $t_{p j}\left(v_{0}, \cdots, v_{p}\right)=\left(v_{0}, \cdots, v_{p}\right)$ if and only if $v_{j}=v_{j+1} \cdot$

(v) The barycentric homomorphism $\beta_{p}: C_{p} \rightarrow C_{p}$ is defined as follows. For integers $p<0, \beta_{p}$ is the trivial homomorphism; for $p=0, \beta_{0}=1$; and for $p>0, \beta_{p}$ is defined by the recursion formula

$$
\beta_{p}\left(v_{0}, \cdots, v_{p}\right)=h_{p-1}^{b} \beta_{p-1} \partial_{p}\left(v_{0}, \cdots v_{p}\right),
$$

where $b$ is the barycenter of the points $v_{0}, \cdots, v_{p}$.

(vi) The barycentric homotopy operator $\rho_{p}$ used by Radó $[1 ; 3, \S 2.2$ (iv); $4, \S 6]$ will not be used in this paper. In its stead, a modification $\rho_{* p}$ is presently introduced, which has a simpler form, satisfies all the important identities which hold for the $\rho_{p}$, and has useful properties not possessed by $\rho_{p}$. The modified barycentric homotopy operator

$$
\rho_{* p}: C_{p} \longrightarrow C_{p+1}
$$

is defined as follows. For integers $p<0, \rho_{* p}$ is the trivial homomorphism; for $p=0, \rho_{* p}$ is defined by the relation

$$
\rho_{*_{0}}\left(v_{0}\right)=-h_{0}^{v_{0}}\left(v_{0}\right)=\left(v_{0}, v_{0}\right) ;
$$

and for $p>0, \rho_{* p}$ is defined by the recursion formula

$$
\rho_{* p}\left(v_{0}, \cdots, v_{p}\right)=-h_{p}^{b}\left[1+\rho_{* p-1} \partial_{p}\right]\left(v_{0}, \cdots, v_{p}\right),
$$

where $b$ is the barycenter of the points $v_{0}, \cdots, v_{p}$.

1.3. Amongst the preceding homomorphisms the following identities hold (see $[2, \S 2 ; 3, \S 2.3])$ :

$$
\begin{array}{lr}
\partial_{p}=\sum_{j=0}^{p} j_{p} & (p \geq 0) ; \\
\partial_{p}+{ }_{1} h_{p}^{v}+h_{p-1}^{v} \partial_{p}=1 & (p>0) ; \\
\partial_{p} \beta_{p}=\beta_{p-1} \partial_{p} & (-\infty<p<+\infty) ; \\
\beta_{p} t_{p j}=-\beta_{p} & (0 \leq j \leq p-1) ; \\
\partial_{p+1} \rho_{* p}+\rho_{* p-1} \partial_{p}=\beta_{p}-1 & (0 \leq p<+\infty) .
\end{array}
$$


Of these identities, only the last is new; it may be established by an inductive reasoning similar to that used to prove the corresponding identity for the conventional barycentric homotopy operator $\rho_{p}$.

1.4. For integers $k, p$ such that $0 \leq k \leq p$, the homomorphism

$$
k_{* p}: C_{p} \rightarrow C_{p}
$$

is defined by the relation

$$
k_{* p}\left(v_{0}, \cdots, v_{p}\right)=(-1)^{p+k}\left(v_{0}, \cdots, \hat{v}_{k}, \cdots, v_{p}, v_{k}\right),
$$

and the homomorphism

$$
\gamma_{p}: C_{p} \longrightarrow C_{p}
$$

is defined by the formula $\gamma_{p}=\sum_{k=0}^{p} k_{* p}$. Obviously one has the identities

$$
\begin{aligned}
& k_{* p}\left(v_{0}, \cdots, v_{p}\right)=-k_{p+1} h_{p}^{v_{k}}\left(v_{0}, \cdots, v_{p}\right), p \geq 0, \\
& k_{* p}\left(v_{0}, \cdots, v_{p}\right)=h_{p-1}^{v_{k}} k_{p}\left(v_{0}, \cdots, v_{p}\right), p>0 .
\end{aligned}
$$

Now the reader will easily verify the relations

$$
\begin{aligned}
j_{p} k_{* p}=\left\{\begin{array}{cl}
(k-1)_{* p-1} j_{p} & , 0 \leq j<k \leq p, \\
k_{* p-1}(j+1)_{p} & , 0 \leq k \leq j<p, \\
k_{p} & , 0 \leq k \leq j=p ;
\end{array}\right. \\
k_{* p-1} j_{p}=\left\{\begin{array}{cl}
(j-1)_{p} k_{* p} & , 0 \leq k<j \leq p, \\
j_{p}(k+1)_{* p} & , 0 \leq j \leq k<p .
\end{array}\right.
\end{aligned}
$$

From these relations the following identity is readily established:

$$
\gamma_{p-1} \partial_{p}=\partial_{p}\left(\gamma_{p}-1\right)
$$

Using the identity, the reader will easily prove the following result.

LEMMA. If $P(x)$ be any polynomial having integral coefficients, then

$$
P\left(\gamma_{p-1}\right) \partial_{p}=\partial_{p} P\left(\gamma_{p}-1\right)
$$

Explicitly, if $P(x)=\sum_{i=0}^{m} a_{i} x^{i}$, where the $a_{i}$ are integers, then 


$$
\sum_{i=0}^{m} a_{i} \gamma_{p-1}^{i} \partial_{p}=\sum_{i=0}^{m} a_{i} \partial_{p}\left[\gamma_{p}^{i}-i \gamma_{p}^{i-1}+\cdots+(-1)^{i}\right]
$$

where $\gamma_{p}^{i}$ means that the homomorphism $\gamma_{p}$ is to be repeated $i$ times.

1.5. For integers $k, p$ such that $0 \leq k \leq p$, the homomorphism

$$
b_{p k}: C_{p} \rightarrow C_{p}+1
$$

is defined by the relation

$$
\begin{aligned}
b_{p k}\left(v_{0}, \cdots, v_{p}\right)=(-1)^{k} & {\left[v_{0}, \cdots, v_{k}, b\left(v_{0}, \ldots, v_{k}\right),\right.} \\
& \left.b\left(v_{0}, \cdots, v_{k}, v_{k}+1\right), \cdots, b\left(v_{0}, \cdots, v_{k}, \cdots, v_{p}\right)\right],
\end{aligned}
$$

where $b\left(v_{0}, \cdots, v_{q}\right)$ is the barycenter of the points $v_{0}, \cdots, v_{q}$. Verification of the following simple relations is left to the reader :

$$
\begin{aligned}
& -h_{p}^{b\left(v_{0}, \cdots, v_{p}\right)}\left(v_{0}, \ldots, v_{p}\right)=b_{p p}\left(v_{0}, \cdots, v_{p}\right) ; \\
& -h_{p}^{b\left(v_{0}, \cdots, v_{p}\right)} b_{p-1 k}\left(v_{0}, \cdots, v_{p-1}\right)=b_{p k} h_{p-1}^{v_{p}}\left(v_{0}, \cdots, v_{p-1}\right) \\
& -h_{p}^{b\left(v_{0}, \cdots, v_{p}\right)} b_{b_{p-1 k}} j_{p}\left(v_{0}, \cdots, v_{p}\right)=b_{p k J * p}\left(v_{0}, \cdots, v_{p}\right) \\
& . \quad(0 \leq k \leq p-1,0 \leq j \leq p) ;
\end{aligned}
$$$$
-h_{p}^{b\left(v_{0}, \cdots, v_{p}\right)} b_{p-1 k} \partial_{p}\left(v_{0}, \cdots, v_{p}\right)=b_{p k} \gamma_{p}\left(v_{0}, \ldots, v_{\mathrm{p}}\right)
$$$$
(0 \leq k \leq p-1)
$$$$
\left\{\begin{array}{r}
-h_{p}^{b\left(v_{0}, \cdots, v_{p}\right)} b_{p-1 k} \partial_{p} \gamma_{p}^{i-1} j_{* p}\left(v_{0}, \cdots, v_{p}\right)=b_{p k} \gamma_{p}^{i} j_{* p}\left(v_{0}, \cdots, v_{p}\right) \\
(0 \leq k \leq p-1,0 \leq j \leq p, 1 \leq i) \\
-h_{p}^{b\left(v_{0}, \cdots, v_{p}\right)} b_{p-1 k} \partial_{p} \gamma_{p}^{i}\left(v_{0}, \cdots, v_{p}\right)=b_{p k} \gamma_{p}^{i+1}\left(v_{0}, \cdots, v_{p}\right) \\
(0 \leq k \leq p-1,0 \leq i) .
\end{array}\right.
$$

If $P(x)$ be any polynomial having integral coefficients, then, for $0 \leq k \leq p-1$, we have

$$
-h_{p}^{b\left(v_{0}, \cdots, v_{p}\right)} b_{p-1 k} \partial_{p} P\left(\gamma_{p}\right)\left(v_{0}, \cdots, v_{p}\right)=b_{p k} \gamma_{p} P\left(\gamma_{p}\right)\left(v_{0}, \ldots, v_{p}\right) .
$$


1.6. For the homomorphisms $\beta_{p}$ and $\rho_{* p} t_{\text {the }}$ following structural descriptions are now obtained.

THE OREM. The following relations hold:

$$
\begin{aligned}
& \rho_{*_{0}}=b_{00}, \\
& \rho_{* p}=b_{p p}+\sum_{j=1}^{p} b_{p p}-j \gamma_{p} \cdots\left(\gamma_{p}-j+1\right) \quad(p>0) .
\end{aligned}
$$

Proof. It is sufficient to verify these formulas for a given $p$-cell $\left(v_{0}, \cdots, v_{p}\right)$. For $p=0$, the formula $\rho_{*_{0}}\left(v_{0}\right)=b_{00}\left(v_{0}\right)$ is obvious from the definitions. So assume that

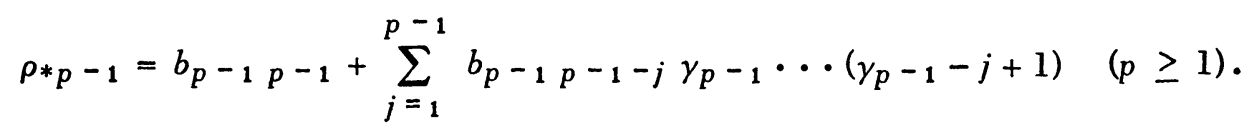

Using $\S 1.2, \S 1.4, \S 1.5$, and this assumption, and letting $b=b\left(v_{0}, \cdots, v_{p}\right)$, one obtains

$$
\begin{aligned}
& \rho_{* p}\left(v_{0}, \cdots, v_{0}\right) \\
& =-h_{p}^{b}\left(v_{0}, \cdots, v_{p}\right)-h_{p}^{b} \rho_{* p-1} \partial_{p}\left(v_{0}, \cdots, v_{p}\right) \\
& =b_{p p}\left(v_{0}, \cdots, v_{p}\right)-h_{p}^{b} b_{p-1 p-1} \partial_{p}\left(v_{0}, \cdots, v_{p}\right) \\
& -\sum_{j=1}^{p-1} h_{p}^{b} b_{p-1 p-1-j} \gamma_{p-1} \cdots\left(\gamma_{p}-1-j+1\right) \partial_{p}\left(v_{0}, \cdots, v_{p}\right) \\
& =b_{p p}\left(v_{0}, \cdots v_{p}\right)+b_{p p}-1 \gamma_{p}\left(v_{0}, \cdots, v_{p}\right) \\
& -\sum_{j=1}^{p-1} h_{p}^{b} b_{p-1 p-1-j} \partial_{p}\left(\gamma_{p}-1\right) \cdots\left(\gamma_{p}-j\right)\left(v_{0}, \cdots, v_{p}\right) \\
& =b_{p p}\left(v_{0}, \cdots, v_{p}\right)+b_{p p-1} \gamma_{p}\left(v_{0}, \ldots, v_{p}\right) \\
& +\sum_{j=2}^{p} b_{p p-j} \gamma_{p}\left(\gamma_{p}-1\right) \cdots\left(\gamma_{p}-j+1\right)\left(v_{0}, \cdots, v_{p}\right) \\
& =b_{p p}+\sum_{j=1}^{p} b_{p p-j} \gamma_{p} \cdots\left(\gamma_{p}-j+1\right)\left(v_{0}, \cdots, v_{p}\right) .
\end{aligned}
$$


So the proof is complete by induction.

1.7. THEOREM. The following relations hold:

$$
\begin{aligned}
& \beta_{0}=0_{1} b_{00}, \\
& \beta_{p}=0_{p+1} b_{p 0} \gamma_{p}\left(\gamma_{p}-1\right) \cdots\left(\gamma_{p}-p+1\right), p>0 .
\end{aligned}
$$

The proof is similar to that for the theorm in the preceding section.

1.8. From these formulas for $\beta_{p}$ and $\rho_{* p}$ and the identities in $\$ 1.3$, many further interesting relations may be obtained. For example, it is easy to establish the following results:

$$
\begin{array}{ll}
\beta_{p}=\left[\partial_{p+1}-(p+1)_{p+1}\right] \rho_{* p} & (p \geq 0) ; \\
\partial_{p}=-p_{p} \rho * p-1 \partial_{p} & (p \geq 0) ; \\
\beta_{p}=(p+1)_{p+1}(p+2)_{p+2} \rho_{* p+1} \rho_{* p} & (p \geq 0) .
\end{array}
$$

These relations are not needed for the present purposes; they may be studied on a later occasion.

In order to clarify the structural descriptions for $\beta_{p}$ and $\rho_{* p}$ given in $\$ \S 1.6$, 1.7 , it is convenient to introduce another homomorphism.

1.9. For integers $p \geq 0$, let $i_{0}, \cdots, i_{p}$ be any rearrangement of the sequence $0, \cdots, p$, and put $\epsilon_{i_{0}} \cdots i_{p}$ equal to +1 or to -1 according as $i_{0}, \cdots, i_{p}$ is obtained from $0, \ldots, p$ by an even or by an odd number of transpositions. With each rearrangement one associates a homomorphism

$$
\tau_{p}: C_{p} \rightarrow C_{p}
$$

defined by the formula

$$
\tau_{p}\left(v_{0}, \cdots, v_{p}\right)=\epsilon_{i_{0}} \cdots i_{p}\left(v_{i_{0}}, \cdots, v_{i_{p}}\right) .
$$

Sometimes, for clarity, the more explicit notation $\tau_{p}\left(i_{0}, \cdots, i_{p}\right)$ is used for this homomorphism. For integers $j$ such that $0 \leq j \leq p$, denote by $T_{p j}$ the class of all $\tau_{p}\left(i_{0}, \cdots, i_{p}\right)$ for which $i_{0}<\cdots<i_{j}$ - that is, for which $i_{0}, \cdots, i_{j}$ are in natural order. Obviously $T_{p p}$ consists of just one element, namely $\tau_{p}(0, \cdots, p)=1$; and $T_{p 0}$ consists of the $\tau_{p}$ obtained by all possible rearrangements of $0, \cdots, p$. Moreover, $T_{p j-1} \supset T_{p j}$ for $1 \leq j \leq p$. Clearly the number of elements in the class $T_{p j}$ is $(p+1) p \cdots(j+2)$ for $0 \leq j \leq p-1$. For each integer $j$ in $0 \leq j \leq p$, define a homomorphism 


$$
P_{p j}: C_{p} \rightarrow C_{p}
$$

by the formula

$$
P_{p j}=\sum \tau_{p}
$$

Observe that $P_{p p}=1$. The reader will readily verify these identities:

$$
\begin{gathered}
k_{* p} P_{p j}=P_{p j}, 0 \leq j<k \leq p ; \\
\sum_{k=0}^{j} k_{* p} P_{p j}=P_{p j-1}, 0<j \leq p .
\end{gathered}
$$

From these identities, the following result is established.

LEMMA. The following relations hold:

$$
\begin{aligned}
P_{p p} & =1, \\
P_{p P-j} & =\gamma_{p}\left(\gamma_{p}-1\right) \cdots\left(y_{p}-j+1\right), 1 \leq j \leq p .
\end{aligned}
$$

Proof. That $P_{p p}=1$ was noted above. From the second relation above it follows that

$$
P_{p p-1}=\sum_{k=0}^{p} k_{* p} P_{p p}=\gamma_{p} P_{p p}=\gamma_{p}
$$

so the general formula is established for $j=1$. Now suppose that

$$
P_{p p-j+1}=\gamma_{p}\left(\gamma_{p}-1\right) \cdots\left(\gamma_{p}-j+2\right) \quad(2 \leq j \leq p) .
$$

Using the preceding identities, one finds

$$
\begin{aligned}
\gamma_{p} P_{p p-j+1} & =\sum_{k=0}^{p} k_{* p} P_{p p-j+1} \\
& =\sum_{k=0}^{p-j+1} k_{* p} P_{p p-j+1}+\sum_{k=p-j+2}^{p} k_{* p} P_{p p-j+1} \\
& =P_{p p-j}+(j-1) P_{p p-j+1} ; \\
P_{p p-j} & =\left(\gamma_{p}-j+1\right) P_{p p-j+1}=\gamma_{p}\left(\gamma_{p}-1\right) \cdots\left(\gamma_{p}-j+1\right) .
\end{aligned}
$$

Thus the lemma is established. 
1.10. Combining the results of the preceding lemma with those in the theoems in $\$ \$ 1.6,1.7$, one obtains the following description for the homomorphisms $\beta_{p}$ and $\rho_{* p}$.

THE OREM. The following relations hold:

$$
\begin{array}{lll}
\beta_{p}=0_{p+1} b_{p 0} P_{p 0}=\sum_{\tau_{p} \in T_{p 0}} 0_{p+1} b_{p 0} \tau_{p} & (p \geq 0) ; \\
\rho_{* p}=\sum_{k=0}^{p} b_{p k} P_{p k}=\sum_{k=0}^{p} \sum_{\tau_{p} \in T_{p k}} b_{p k} \tau_{p} & (p \geq 0) .
\end{array}
$$

1.11. Let $v_{0}, \cdots, v_{p}(p \geq 0)$ be any sequence of $p+1$ points in $E_{\infty}$. In $\S \S 1.2,1.4,1.5,1.9$, homomorphisms $j_{p}, t_{p j}, k_{* p}, b_{p k}, \tau_{p}$, have been introduced which, when applied in any appropriate combination $h_{p}$ to the special chain $\left(v_{0}, \cdots, v_{p}\right)$, yield a special chain either of the form $+\left(y_{0}, \cdots, y_{q}\right)$ or of the form $-\left(y_{0}, \cdots, y_{q}\right)$. In the sequel, $\left[h_{p}\left(v_{0}, \cdots, v_{p}\right)\right]$ is defined to be the $p$-cell $\left(y_{0}, \cdots, y_{q}\right)$, and $\left|h_{p}\left(v_{0}, \cdots, v_{p}\right)\right|$ denotes its convex hull $\left|y_{0}, \cdots, y_{q}\right|$. For example,

$$
\begin{aligned}
{\left[0_{p}+1\right.} & \left.b_{p 0} \tau_{p}\left(i_{0}, \cdots, i_{p}\right)\left(v_{0}, \cdots, v_{p}\right)\right] \\
& =\left(b\left(v_{i_{0}}\right), b\left(v_{i_{0}}, v_{i_{1}}\right), \cdots, b\left(v_{i_{0}}, v_{i_{1}}, \cdots, v_{i_{p}}\right)\right) .
\end{aligned}
$$

If for two sequences of points $u_{0}, \cdots, u_{p}$ and $v_{0}, \cdots, v_{p}$ it is true that

$$
\begin{aligned}
\left(b\left(u_{0}\right), b\left(u_{0}, u_{1}\right), \cdots, b\right. & \left.\left(u_{0}, u_{1}, \cdots, u_{p}\right)\right) \\
& =\left(b\left(v_{0}\right), b\left(v_{0}, v_{1}\right), \cdots, b\left(v_{0}, v_{1}, \cdots, v_{p}\right)\right)
\end{aligned}
$$

then clearly $u_{j}=v_{j}$ for $0 \leq j \leq p$. From the remarks in $\$ 1.9$ and the preceding theorem, one thus obtains the following result.

LEMMA. If the points $v_{0}, \cdots, v_{p}(p \geq 0)$ are distinct, then the chain $\beta_{p}\left(v_{0}, \cdots, v_{p}\right)$ contains $(p+1)$ ! terms; that is, for distinct elements $\tau_{p}^{\prime}$ and $\tau_{p}^{\prime \prime}$ in $T_{p 0}$, we have

$$
\left[0_{p+1} b_{p 0} \tau_{p}^{\prime}\left(v_{0}, \cdots, v_{p}\right)\right] \neq\left[\begin{array}{lll}
0_{p}+1 & b_{p 0} & \left.\tau_{p}^{\prime \prime}\left(v_{0}, \cdots, v_{p}\right)\right]
\end{array}\right.
$$

1.12. LEMmA. Let $v_{0}, \cdots, v_{p}(p \geq 0)$ be any set of $p+1$ points in $E_{\infty}$, 
not necessarily distinct or linearly independent. A necessary and sufficient condition that a point $v$ belong to the convex hull of the points

(i) $b\left(v_{0}\right), b\left(v_{0}, v_{1}\right), \cdots, b\left(v_{0}, v_{1}, \cdots, v_{p}\right)$

is that it possess a representation of the form

(ii) $v=\sum_{j=0}^{p} \mu_{j} v_{j} \quad\left(\sum_{j=0}^{p} \mu_{j}=1, \mu_{0} \geq \mu_{1} \geq \cdots \geq \mu_{p} \geq 0\right)$.

Proof. If $v$ belongs to the convex hull of the points (i), then it has a representation of the form

(iii) $v=\sum_{i=0}^{p} \lambda_{i} b\left(v_{0}, \cdots, v_{i}\right) \quad\left(\sum_{i=0}^{p} \lambda_{i}=1,0 \leq \lambda_{i}, 0 \leq i \leq p\right)$.

Thus

$$
v=\sum_{i=0}^{p} \lambda_{i} \sum_{j=0}^{i} \frac{v_{j}}{i+1}=\sum_{j=0}^{p} \sum_{i=j}^{p} \frac{\lambda_{i}}{i+1} v_{j}
$$

which gives a representation of form (ii) for $v$. Conversely, if $v$ has a representation of form (ii), put $\lambda_{i}=(i+1)\left(\mu_{i}-\mu_{i+1}\right)$ for $0 \leq i \leq p-1, \lambda_{p}=(p+1) \mu_{p}$. It follows at once that $v$ has a representation of form (iii), and hence belongs to the convex hull of the set of points (i).

1.13. For integers $p \geq 0$, if $u_{0}, \cdots, u_{p}$ is any sequence of $p+1$ points in $E_{\infty}$, then $\left|u_{0}, \cdots, u_{p}\right|$ will denote its convex hull. Let $k$ be any integer such that $0 \leq k \leq p$, and consider the sequence of $p+2$ points

(i) $u_{0}, \cdots u_{k}, b\left(u_{0}, \cdots u_{k}\right), \cdots, b\left(u_{0}, \cdots, u_{k}, \cdots, u_{p}\right)$,

that is (see $\$ 1.5)$, the sequence of points occurring in $b_{p k}\left(u_{0}, \ldots, u_{p}\right)$. Let

(ii) $w_{0}, \cdots, w_{p}+1$

be any rearrangement of the sequence of points (i). Designate by $x_{0}=w_{h_{0}}=u_{i_{0}}$ the first $u_{i}(0 \leq i \leq k)$ occurring in the sequence (ii). In general, let $x_{l}=w_{h_{l}}$ $=u_{i_{l}}(0 \leq l \leq k)$ be the $(l+1)$ st $u_{i}(0 \leq i \leq k)$ occurring in the sequence (ii), and put $x_{l}=u_{l}$ for $k+1 \leq l \leq p$ in case $k<p$. Now clearly $x_{0}, \cdots, x_{p}$ is a rearrangement of the sequence $u_{0}, \cdots, u_{p}$ in which the last $p-k$ elements are unaltered; the sequence $(i)$ is a rearrangement of the sequence 
(iii) $x_{0}, \cdots, x_{k}, b\left(x_{0}, \cdots, x_{k}\right), \cdots, b\left(x_{0}, \cdots, x_{k}, \cdots, x_{p}\right)$ in which the last $p+1-k$ elements are unaltered; and the sequence (ii) is a rearrangement of the sequence (iii) in which the points $x_{0}, \ldots, x_{k}$ appear in the same order as in (iii); that is, $x_{l}=w_{h_{l}}$ for $0 \leq l \leq k$, where $0 \leq h_{0}<h_{1}$ $<\ldots<h_{k} \leq p$. Now let $q$ be any integer such that $0 \leq q \leq p+1$. It will be shown that

$$
\text { (iv) } \begin{aligned}
b\left(w_{0}, \cdots, w_{q}\right) \in \mid b\left(x_{0}\right), b\left(x_{0}, x_{1}\right), \cdots, b\left(x_{0}, x_{1},\right. & \left.\cdots, x_{p}\right) \mid \\
& (0 \leq q \leq p+1) .
\end{aligned}
$$

Case $q=0$. Then $b\left(w_{0}\right)=w_{0}$. If $w_{0}$ is one of the $u_{i}(0 \leq i \leq k)$, it follows by the choice above that $h_{0}=0$ and $w_{0}=x_{0}=b\left(x_{0}\right)$. If $w_{0}$ is not one of the $u_{i}(0 \leq i \leq k)$, there must be a $l \geq k$ such that $w_{0}=b\left(u_{0}, \cdots, u_{k}, \cdots, u_{l}\right)$ $=b\left(x_{0}, \cdots, x_{k}, \cdots, x_{l}\right)$. Thus relation (iv) is established when $q=0$.

General case. By a rearrangement, the points $w_{0}, \ldots, w_{q}$ may be ordered into two sets

$$
\begin{gathered}
w_{h_{0}}=x_{0}, \cdots, w_{h_{l}}=x_{l} \quad\left(0 \leq l \leq k, 0 \leq h_{0}<\ldots<h_{l} \leq p\right), \\
\left\{\begin{array}{c}
w_{h_{l+1}}=b\left(u_{0}, \cdots, u_{k}, \cdots, u_{i_{l+1}}\right)=b\left(x_{0}, \cdots, x_{i_{l+1}}\right) \\
w_{h_{l+2}}=b\left(u_{0}, \cdots, u_{k}, \cdots, u_{i_{l+2}}\right)=b\left(x_{0}, \cdots, x_{i_{l+2}}\right) \\
\cdots \\
w_{h_{q}}=b\left(u_{0}, \cdots, u_{k}, \cdots, u_{i_{q}}\right)=b\left(x_{0}, \cdots, x_{i_{q}}\right) \\
\left(k \leq i_{l+1}<i_{l+2}<\ldots<i_{q} \leq p\right) .
\end{array}\right.
\end{gathered}
$$

The special cases which arise when one of these sets is missing are left to the reader. Now clearly

$$
\begin{aligned}
& b\left(w_{0}, \cdots, w_{q}\right)=b\left(w_{h_{0}}, \cdots, w_{h_{q}}\right) \\
& =\sum_{j=0}^{l} \frac{1}{q+1}\left[1+\sum_{h=l+1}^{q} \frac{1}{i_{h}+1}\right] x_{j}+\sum_{j=l+1}^{i_{l+1}} \frac{1}{q+1} \sum_{h=l+1}^{q} \frac{1}{i_{h}+1} x_{j} \\
& +\sum_{j=i_{l+1}+1}^{i_{l+2}} \frac{1}{q+1} \sum_{h=l+2}^{q} \frac{1}{i_{h}+1} x_{j}+\cdots+\sum_{j=i_{q-1}+1}^{i_{q}} \frac{1}{q+1} \frac{1}{i_{q}+1} x_{j} .
\end{aligned}
$$


In view of this equation and of the lemma in $\$ 1.12$, the relation (iv) now follows.

1.14. From the facts presented above, the following result is presently established.

Lemma. Let $v_{0}, \cdots, v_{p}(p \leq 0)$ be any sequence of $p+1$ points in $E_{\infty}$. Fix $\tau_{p+1} \in T_{p+10}(0 \leq k \leq p), \tau_{p} \in T_{p k}$ (see \$1.9). Then there exists $a$ $\tau_{p}^{\prime} \in T_{p 0}$ such that (see $\left.\S 1.11\right)$.

$$
\left|0_{p+2} b_{p+10} \tau_{p+1} b_{p k} \tau_{p}\left(v_{0}, \cdots, v_{p}\right)\right| \subset\left|0_{p+1} b_{p 0} \tau_{p}^{\prime}\left(v_{0}, \cdots, v_{p}\right)\right| .
$$

Proof. Evidently $\left[\tau_{p}\left(v_{0}, \cdots, v_{p}\right)\right]=\left(v_{i_{0}}, \cdots, v_{i_{p}}\right)$, where $i_{0}, \cdots, i_{p}$ is a rearrangement of $0, \cdots, p$ such that $i_{0}<\cdots<i_{k}$. Put $u_{j}=v_{i_{j}}$ for $0 \leq j \leq p$, so that $\left[\tau_{p}\left(v_{0}, \cdots, v_{p}\right)\right]=\left(u_{0}, \cdots, u_{p}\right)$. Then

$$
\begin{aligned}
& {\left[b_{p k} \tau_{p}\left(v_{0}, \cdots, v_{p}\right)\right]} \\
& \quad=\left(u_{0}, \cdots, u_{k}, b\left(u_{0}, \cdots u_{k}\right), \cdots, b\left(u_{0}, \cdots, u_{k}, \cdots, u_{p}\right)\right),
\end{aligned}
$$

and $\left[\tau_{p+1} b_{p k} \tau_{p}\left(v_{0}, \cdots, v_{p}\right)\right]=\left(w_{0}, \cdots, w_{p+1}\right)$, where $w_{0}, \cdots, w_{p+1}^{\oplus}$ is a rearrangement of

$$
u_{0}, \cdots, u_{k}, b\left(u_{0}, \cdots, u_{k}\right), \cdots, b\left(u_{0}, \cdots, u_{k}, \cdots, u_{p}\right) .
$$

Finally,

$$
\begin{aligned}
& {\left[\begin{array}{llllll}
0_{p+2} & b_{p+1} & 0 & \tau_{p+1} & b_{p k} & \tau_{p}\left(v_{0}, \cdots, v_{p}\right)
\end{array}\right]} \\
& =\left[b\left(w_{0}\right), b\left(w_{0}, w_{1}\right), \cdots, b\left(w_{0}, w_{1}, \cdots, w_{p+1}\right)\right] .
\end{aligned}
$$

The reasoning of $\$ 1.13$ shows that there is a rearrangement $x_{0}, \cdots, x_{p}$ of $u_{0}, \cdots, u_{p}$, and hence of $v_{0}, \cdots, v_{p}$, such that

$$
\begin{aligned}
\mid 0_{p+2} b_{p+10} \tau_{p+1} b_{p k} & \tau_{p}\left(v_{0}, \ldots, v_{p}\right) \mid \\
& \subset\left|b\left(x_{0}\right), b\left(x_{0}, x_{1}\right), \ldots, b\left(x_{0}, x_{1}, \ldots, x_{p}\right)\right| .
\end{aligned}
$$

Let $\tau_{p}^{\prime}$ be that element of $T_{p 0}$ such that $\left[\tau_{p}^{\prime}\left(v_{0}, \cdots, v_{p}\right)\right]=\left(x_{0}, \cdots, x_{p}\right)$. Since

$$
\left[0_{p+1} b_{p 0} \tau_{p}^{\prime}\left(v_{0}, \cdots, v_{p}\right)\right]=\left(b\left(x_{0}\right), b\left(x_{0}, x_{1}\right), \ldots, b\left(x_{1}, x_{1}, \ldots, x_{p}\right)\right),
$$

the lemma is established.

1.15. If $c_{p}$ is a $p$-chain in $K$, and $A$ is a convex subset in $E_{\infty}$, then the in- 
clusion $c_{p} \subset A$ will mean that either $c_{p}=0 \in C_{p}$ or else

$$
c_{p}=\sum_{j=1}^{n} m_{j}\left(v_{0 j}, \cdots, v_{p j}\right),
$$

where the $m_{j}$ are nonzero integers and $\left|v_{0 j}, \cdots, v_{p j}\right| \subset A$ for $1 \leq j \leq n$. One readily verifies the following inclusions (see $[3, \S 2.4]$ ):

$$
\begin{array}{rr}
j_{p}\left(v_{0}, \cdots, v_{p}\right) \subset\left|v_{0}, \cdots, v_{p}\right| & (0 \leq j \leq p), \\
\partial_{p}\left(v_{0}, \cdots, v_{p}\right) \subset\left|v_{0}, \cdots, v_{p}\right| & (p \geq 0), \\
\beta_{p}\left(v_{0}, \cdots, v_{p}\right) \subset\left|v_{0}, \cdots, v_{p}\right| & (p \geq 0), \\
\rho_{* p}\left(v_{0}, \cdots, v_{p}\right) \subset\left|v_{0}, \cdots, v_{p}\right| & (p \geq 0), \\
t_{p j}\left(v_{0}, \cdots, v_{p}\right) \subset\left|v_{0}, \cdots, v_{p}\right| & (0 \leq j \leq p-1), \\
k_{* p}\left(v_{0}, \cdots, v_{p}\right) \subset\left|v_{0}, \cdots, v_{p}\right| & (0 \leq k \leq p), \\
\gamma_{p}\left(v_{0}, \cdots, v_{p}\right) \subset\left|v_{0}, \cdots, v_{p}\right| & (p \geq 0), \\
b_{p k}\left(v_{0}, \cdots, v_{p}\right) \subset\left|v_{0}, \cdots, v_{p}\right| & (0 \leq k \leq p), \\
\tau_{p}\left(v_{0}, \cdots, v_{p}\right) \subset\left|v_{0}, \cdots, v_{p}\right| & \left(\tau_{p} \in T_{p 0}\right), \\
P_{p j}\left(v_{0}, \cdots, v_{p}\right) \subset\left|v_{0}, \cdots, v_{p}\right| & (0 \leq j \leq p) .
\end{array}
$$

II. Relations in the Complex $R=R(X)$.

2.1. If $A$ is a convex subset of $E_{\infty}$, then for integers $p \geq 0, C_{p}^{A}$ denotes that subgroup of $C_{p}$ generated by those $p$-cells $\left(v_{0}, \cdots, v_{p}\right)$ for which $\left|v_{0}, \cdots, v_{p}\right|$ $\subset A$; for $p<0$, we have $C_{p}^{A}=0 \in C_{p}$ (see $\$ 1.1$ ). Suppose $T: A \rightarrow X$ is a continuous mapping (see $\S 0.1$ ). For integers $p \geq 0$ define a homomorphism

$$
T_{p}: C_{p}^{A} \rightarrow C_{p}^{R}
$$

by the relation $T_{p}\left(v_{0}, \cdots, v_{p}\right)=\left(v_{0}, \cdots, v_{p}, T\right)^{R}$ for $\left(v_{0}, \cdots, v_{p}\right) \in C_{p}^{A}$. For $p<0$, let $T_{p}$ be the trivial homomorphism. For chains $c_{p}$ in $C_{p}^{A}$ the notation $T_{p} c_{p}=\left(c_{p}, T\right)^{R}$ is used. In terms of this notation one finds the relation (see $\S 0.1): \partial_{p}^{R}\left(c_{p}, T\right)^{R}=\left(\partial_{p} c_{p}, T^{\prime}\right)^{R}$.

Now suppose that, for certain integers $p$,

$$
h_{p}: C_{p} \longrightarrow C_{q}
$$

is a homomorphism from the group $C_{p}$ of $p$-chains into the group $C_{q}$ of $q$-chains 
in $K$ with the property that for all $p$-cells $\left(v_{0}, \cdots, v_{p}\right)$ in $K$ one has

$$
h_{p}\left(v_{0}, \cdots, v_{p}\right) \subset\left|v_{0}, \cdots, v_{p}\right| \text {. }
$$

Then clearly one may define for these integers $p$ a homomorphism

$$
h_{p}^{R}: C_{p}^{R} \rightarrow C_{q}^{R}
$$

by the formula $h_{p}^{R}\left(v_{0}, \cdots, v_{p}, T\right)^{R}=\left(h_{p}\left(v_{0}, \ldots, v_{p}\right), T\right)^{R}$ in case $p \geq 0$, and one may make $h_{p}^{R}$ the trivial homomorphism if $p<0$. In view of the inclusions in $\S 1.15$, one observes that this definition creates the following homomorphisms in $R($ see $[3, \S 3.1])$ :

$$
\begin{array}{cll}
j_{p}^{R}: C_{p}^{R} \rightarrow C_{p-1}^{R} \quad(0 \leq j \leq p) ; & \\
\beta_{p}^{R}: C_{p}^{R} \rightarrow C_{p}^{R} \quad(-\infty<p<+\infty) ; & \gamma_{p}^{R}: C_{p}^{R} \rightarrow C_{p}^{R} \quad(p \geq 0) ; \\
\rho_{* p}^{R}: C_{p}^{R} \rightarrow C_{p+1}^{R} \quad(-\infty<p<+\infty) ; & b_{p k}^{R}: C_{p}^{R} \rightarrow C_{p+1}^{R} \quad(0 \leq k \leq p) ; \\
t_{p j}^{R}: C_{p}^{R} \rightarrow C_{p-1}^{R}(0 \leq j \leq p-1) ; & \tau_{p}^{R}: C_{p}^{R} \rightarrow C_{p}^{R} \quad\left(\tau_{p} \in T_{p_{0}}\right) ; \\
& P_{p j}^{R}: C_{p}^{R} \rightarrow C_{p}^{R} \quad(0 \leq j \leq p) .
\end{array}
$$

2.2. From the relations in $\S 1.3$, one derives the following (see $[3, \S 3.1]$ ):

$$
\begin{array}{ll}
\partial_{p}^{R} \beta_{p}^{R}=\beta_{p-1}^{R} \partial_{p}^{R} & (-\infty<p<+\infty) ; \\
\beta_{p}^{R} t_{p j}^{R}=-\beta_{p}^{R} & (0 \leq j \leq p-1) ; \\
\partial_{p+1}^{R} \rho_{* p}^{R}+\rho_{{ }^{*} p-1}^{R} \partial_{p}^{R}=\beta_{p}^{R}-1 & (0 \leq p<+\infty) .
\end{array}
$$

The theorems in $\S \S 1.6,1,7$ give rise to these formulas for $\beta_{p}^{R}$ and $\rho_{* p}^{R}$ :

$$
\begin{aligned}
& \rho_{* 0}^{R}=b_{00}^{R}, \\
& \rho_{* p}^{R}=b_{p p}^{R}+\sum_{j=1}^{p} b_{p p-j}^{R} \gamma_{p}^{R}, \cdots,\left(\gamma_{p}^{R}-j+1\right)(p>0) ; \\
& \beta_{0}^{R}=0_{p}^{R} b_{00}^{R} ; \\
& \beta_{p}^{R}=0_{p+1}^{R} b_{p 0}^{R} \gamma_{p}^{R}\left(\gamma_{p}^{R}-1\right), \cdots,\left(\gamma_{p}^{R}-p+1\right)(p>0) .
\end{aligned}
$$

From the theorem in $\$ 1.10$, one obtains the following description for $\beta_{p}^{R}$ and $\rho_{* p}^{R}$. 
THEOREM. The following relations hold:

$$
\begin{aligned}
& \beta_{p}^{R}=0_{p+1}^{R} b_{p 0}^{R} P_{p 0}^{R}=\sum_{\tau_{p}} \sum_{T_{p 0}} 0_{p+1}^{R} b_{p 0}^{R} \tau_{p}^{R}(p \geq 0) ; \\
& \rho_{* p}^{R}=\sum_{k=0}^{p} b_{p k}^{R} P_{p k}^{R}=\sum_{k=0}^{p} \sum_{\tau_{p}} \underset{\epsilon}{\in} b_{p k} b_{p k}^{R} \tau_{p}^{R}(p \geq 0) .
\end{aligned}
$$

2.3. The writer is indebted to T. Radó for suggestions which led to the results presently presented in $\$ \S 2.3-2.7,2.9,2.10,2,12$. The new facts contributed by this paper are contained in $\$ \$ 2.8,2.11,2.13$. For integers $p \geq 1$, any chain of the form $\left(1+t_{p i}^{R}\right)\left(v_{0}, \cdots, v_{p}, T\right)^{R}(0 \leq j \leq p-1)$ is termed an elementary $t$-chain in $R$ (see $[3, \S 3.2]$ or $[4, \S 7]$ ), and the subgroup of $C_{p}^{R}$ generated by these elementary $t$-chains is denoted by $T_{p}^{R}$. For $p<1, T_{p}^{R}$ is defined to be the subgroup of $C_{p}^{R}$ composed of the zero element alone.

LEMma. If $c_{p}^{R} \in T_{p}^{R}$, then

(i) $\partial_{p}^{R} c_{p}^{R} \in T_{p-1}^{R}$,

(ii) $\beta_{p}^{R} c_{p}^{R}=0$,

(iii) $\rho_{* p}^{R} c_{p}^{R} \in T_{p+1}^{R}$.

This lemma differs from that in Rado [3, $\$ 3.2]$, only by the fact that the barycentric homotopy operator $\rho_{p}^{R}$ has been replaced by the modified operator $\rho_{* p}^{R}$ (see $\$ 1.2$ ). It may be established by the same reasoning as that employed by Radó.

2.4. For integers $p \geq 1$, any chain of the form

$$
\left(v_{0}, \cdots, v_{j}, v_{j+1}, \cdots, v_{p}, T\right)^{R}
$$

with $v_{j}=v_{j+1}$ for some $j$ such that $0 \leq j \leq p-1$ is called an elementary $d$-chain in $R$ (see $[3, \$ 3.3]$ or $[4, \$ 7]$ ), and the subgroup of $C_{p}^{R}$ generated by these elementary $d$-chains is denoted by $D_{p}^{R}$. For $p<1, D_{p}^{R}$ is defined to be that subgroup of $C_{p}^{R}$ composed of the zero element alone.

LEMma. If $c_{p}^{R} \in D_{p}^{R}$, then

(i) $\partial_{p}^{R} c_{p}^{R} \in D_{p-1}^{R}$,

(ii) $\beta_{p}^{R} c_{p}^{R}=0$, 
(iii) $\rho_{* p}^{R} c_{p}^{R} \in D_{p+1}^{R}$.

This is the lemma in $[3, \S 3.3]$, except that the modified barycentric homotopy operator $\rho_{* p}^{R}$ is used in place of $\rho_{p}^{R}$; it is proved in the same way.

2.5. LemMA. Let $\left(v_{0}, \cdots, v_{p}, T\right)^{R}$ be any $p$-cell in $R(p \geq 1)$. Suppose that the sequence $w_{0}, \cdots, w_{p}$ is obtainable from the sequence $v_{0}, \cdots, v_{p}$ by $n$ transpositions. Then there is an element $t_{p}^{R}$ in $T_{p}^{R}$ such that

$$
\left(v_{0}, \cdots, v_{p}, T\right)^{R}=(-1)^{n}\left(w_{0}, \cdots, w_{p}, T\right)^{R}+t_{p}^{R} .
$$

Proof. By assumption there exist $n+1$ sequences $v_{0 j}, \cdots, v_{p j}$ for $0 \leq j \leq n$ where $v_{i 0}=v_{i}$ and $v_{i n}=w_{i}$ for $0 \leq i \leq p$ such that

$$
\left(v_{0 j}, \cdots, v_{p j}, T\right)^{R}=t_{p i_{j}}^{R}\left(v_{0 j-1}, \cdots, v_{p j-1}, T\right)^{R}
$$

for some integer $i_{j}$ satisfying $0 \leq i_{j} \leq p-1,1 \leq j \leq n$. Clearly

$$
\begin{aligned}
\left(v_{0}, \cdots, v_{p}, T\right)^{R}=(-1)^{n}\left(w_{0}, \cdots, w_{p}, T\right)^{R} \\
\quad+\sum_{j=1}^{n}(-1)^{j-1}\left(1+t_{p i_{j}}^{R}\right)\left(v_{0 j-1}, \cdots, v_{p j-1}, T\right)^{R},
\end{aligned}
$$

and the lemma is established.

2.6. Lemma. Let $\left(v_{0}, \cdots, v_{p}, T\right)^{R}$ be any $p$-cell in $R(p \geq 1)$, for which $v_{i}=v_{k}$ for some $i, k$ such that $0 \leq i \leq k \leq p$. Then there are elements $t_{p}^{R}$ in $T_{p}^{R}$ and $d_{p}^{R}$ in $D_{p}^{R}$ such that

$$
\left(v_{0}, \cdots, v_{p}, T\right)^{R}=t_{p}^{R}+d_{p}^{R}
$$

Moreover, $2\left(v_{0}, \cdots, v_{p}, T\right)^{R}$ is in $T_{p}^{R}$.

Proof. Since the sequence $v_{0}, \cdots, v_{i-1}, v_{k}, v_{i}, \cdots, v_{k-1}, v_{k+1}, \cdots, v_{p}$ is obtained from $v_{0}, \cdots, v_{i}, \cdots, v_{k}, \cdots, v_{p}$ by $k-i$ transpositions, and $v_{i}=v_{k}$ by assumption, if follows that

$$
(-1)^{k-i}\left(v_{0}, \cdots, v_{i-1}, v_{k}, v_{i}, \cdots, v_{k-1}, v_{k+1}, \cdots, v_{p}\right)^{R}
$$

is an element $d_{p}^{R}$ of $D_{p}^{R}$. Moreover, from the lemma in $\$ 2.5$ it follows that there is an element $t_{p}^{R}$ in $T_{p}^{R}$ such that $\left(v_{0}, \cdots, v_{p}, T\right)^{R}=d_{p}^{R}+t_{p}^{R}$, and the first part of the lemma is proven. Now the sequence $v_{0}, \cdots, v_{k}, \cdots, v_{i}, \cdots, v_{p}$ is obtained from $v_{0}, \cdots, v_{i}, \cdots, v_{k}, \cdots, v_{p}$ by $2(k-i)-1$ transpositions. 
Again, from the lemma in $\S 2.5$ it follows that there is an element $t_{p}^{R}$ in $T_{p}^{R}$ such that

$$
\left(v_{0}, \cdots, v_{i}, \cdots, v_{k}, \cdots, v_{p}, T\right)^{R}=-\left(v_{0}, \cdots, v_{k}, \cdots, v_{i}, \cdots, v_{p}, T\right)^{R}+t_{p}^{R} .
$$

Since $v_{i}=v_{k}$, one obtains $2\left(v_{0}, \cdots, v_{p}, T\right)^{R}=t_{p}^{R}$; and the second part of the lemma is demonstrated.

2.7. For integers $p \geq 0$, a chain $c_{p}^{R}$ is termed an elementary $n$-chain in $R$ if it has the form

$$
c_{p}^{R}=\sum_{r=1}^{n} m_{r}\left(v_{0}, \cdots, v_{p}, T_{r}\right)^{R}
$$

where

(i) for $1 \leq r \leq n$, the $m_{r}$ are nonzero integers;

(ii) for $1 \leq r_{1}<r_{2} \leq n$, the transformations $T_{r_{1}}$ and $T_{r_{2}}$ are not identical on $\left|v_{0}, \cdots, v_{p}\right|$;

(iii) the points $v_{0}, \cdots, v_{p}$ are distinct. The $p$-cell $\left(v_{0}, \cdots, v_{p}\right)$ in $K$ (see $\S 1.11)$ is called the base for $c_{p}^{R}$, and the notation $c_{p}^{R}=c_{p}^{R}\left(v_{0}, \cdots, v_{p}\right)$ is used when it is desirable to display the base.

2.8. Lemma. Suppose that $c_{p}^{R}$ is an elementary n-chain in $R$ for which $\beta_{p}^{R} c_{p}^{R}=0$. Then $\beta_{p+1}^{R} \rho_{* p}^{R} c_{p}^{R}=0$.

Proof. With the notation of $\$ 2.7$, one finds (see $\$ \S 2.1,2.2$ ).

(i) $\beta_{p}^{R} c_{p}^{R}=\sum_{\tau_{p} \in T_{p 0}} \sum_{r=1}^{n} m_{r}\left(0_{p+1} b_{p 0} \tau_{p}\left(v_{0}, \cdots, v_{p}\right), T_{r}\right)^{R}=0$;

(ii) $\beta_{p+1}^{R} \rho_{*_{p}}^{R} c_{p}^{R}=\sum_{\tau_{p+1}} \sum_{T_{p+1} 0} \sum_{k=0}^{p} \sum_{\tau_{p} \in T_{p k}} \sum_{r=1}^{n} m_{r}\left(0_{p+2} b_{p+10}\right.$ $\left.\tau_{p+1} b_{p k} \tau_{p}\left(v_{0}, \cdots, v_{p}\right), T_{r}\right)^{R}$.

In view of $\$ 2.7$ (iii), and $\S 1.11$, it follows from (i) that for each $\tau_{p}^{\prime} \in T_{p 0}$, one has

$$
\text { (iii) } \sum_{r=1}^{n} m_{r}\left(0_{p+1} b_{p 0} \tau_{p}^{\prime}\left(v_{0}, \cdots, v_{p}\right), T_{r}\right)^{R}=0 \quad\left(\tau_{p}^{\prime} \in T_{p 0}\right) \text {, }
$$


Fix

$$
\tau_{p+1} \in T_{p+10}, \tau_{p} \in T_{p k} \quad(0 \leq k \leq p) .
$$

From the lemma in $\$ 1.14$ follows the existence of a $\tau_{p}^{\prime} \in T_{p 0}$ such that

(iv) $\left|0_{p+2} b_{p+10} \tau_{p+1} b_{p k} \tau_{p}\left(v_{0}, \ldots, v_{p}\right)\right|$

$$
\subset\left|0_{p+1} b_{p 0} \tau_{p}^{\prime}\left(v_{0}, \cdots, v_{p}\right)\right| \text {. }
$$

From (iii) and (iv) one concludes that for each

$$
\tau_{p+1} \in T_{p+10}, \tau_{p} \in T_{p k} \quad(0 \leq k \leq p),
$$

we have

$$
\text { (v) } \sum_{r=1}^{n} m_{r}\left(0_{p+2} b_{p+10} \tau_{p+1} b_{p k} \tau_{p}\left(v_{0}, \cdots, v_{p}\right), T\right)^{R}=0 \text {. }
$$

In view of (ii) and (v) the lemma is now established.

2.9. For integers $p \geq 0$, the class $N_{p}^{R}$ is defined to be that subset of $C_{p}^{R}$ composed of the chain $0 \bar{\epsilon} C_{p}^{R}$ and of all $c_{p}^{R}$ having a representation of the form

$$
c_{p}^{R}=\sum_{s=1}^{n} c_{p s}^{R}\left(v_{0 s}, \cdots, v_{p s}\right)
$$

where

(i) for $1 \leq s \leq n$ the $c_{p s}^{R}\left(v_{0 s}, \cdots, v_{p s}\right)$ are elementary $n$-chains (see 2.7);

(ii) for $1 \leq s_{1}<s_{2} \leq n$, the point sets $v_{0 s_{1}}, \cdots, v_{p s_{1}}$ and $v_{0 s_{2}}, \cdots, v_{p s_{2}}$ are distinct. For $p<0$, the class $N_{p}^{R}$ consists of the chain $0 € C_{p}^{R}$ alone. Each of the elementary $n$-chains $c_{p s}^{R}\left(v_{0 s}, \cdots, v_{p s}\right)(1 \leq s \leq n)$, is termed a $n$-composant of $c_{p}^{R}$. Observe that the sets $N_{p}^{R}$ are not generally subgroups of $C_{p}^{R}$

2.10. Lemma. Let

$$
c_{p}^{R}=\sum_{s=1}^{n} c_{p s}^{R}\left(v_{0 s}, \cdots, v_{p s}\right)
$$

be any nonzero element in $N_{p}^{R}$. A necessary and sufficient condition in order that 
$\beta_{p}^{R} c_{p}^{R}=0$ is that $\beta_{p}^{R} c_{p s}^{R}=0$ for every $n$-composant $c_{p s}^{R}(1 \leq s \leq n)$.

Proof. Trivially the condition suffices. It is presently shown to be necessary. With explicit notations (see $\$ \S 2.7,2.9$ ),

$$
\begin{aligned}
\beta_{p}^{R} c_{p}^{R} & =\sum_{s=1}^{n} \beta_{p}^{R} c_{p s}^{R}=\sum_{s=1}^{n} \sum_{r=1}^{n_{s}} m_{r s}\left(\beta_{p}\left(v_{0 s}, \cdots, v_{p s}\right) T_{r s}\right)^{R} \\
& =\sum_{s=1}^{n} \sum_{r=1}^{n_{s}} \sum_{\tau_{p} \in T_{p 0}} m_{r s}\left(0_{p+1} b_{p 0} \tau_{p}\left(v_{0 s}, \cdots, v_{p s}\right), T_{r s}\right)^{R}=0 .
\end{aligned}
$$

In view of $\$ 2.9$ (ii) and of the remarks in $\$ 1.11$, it is clear (see $\$ 0.2)$ that, for $1 \leq s \leq n$ we have

$$
\beta_{p}^{R} c_{p s}^{R}=\sum_{r=1}^{n_{s}} \sum_{\tau_{p} \in T_{p 0}} m_{r s}\left(0_{p}+1 b_{p 0} \tau_{p}\left(v_{0 s}, \cdots, v_{p s}\right), T_{r s}\right)^{R}=0
$$

and hence the assertion in the lemma is verified.

2.11. Lemma. Let $c_{p}^{R}$ be any element in $N_{p}^{R}$ for which $\beta_{p}^{R} c_{p}^{R}=0$. Then

$$
\beta_{p+1}^{R} \rho_{* p}^{R} c_{p}^{R}=0 \text {. }
$$

This result is an immediate consequence of the lemmas in $\$ \$ 2.8,2.10$.

2.12. LemmA. Every chain $c_{p}^{R}$ has a representation of the form (see $\$ \$ 2.3$, $2.4,2.9)$

$$
c_{p}^{R}=t_{p}^{R}+d_{p}^{R}+n_{p}^{R} \quad\left(t_{p}^{R} \in T_{p}^{R}, d_{p}^{R} \in D_{p}^{R}, n_{p}^{R} \in N_{p}^{R}\right) .
$$

Generally this representation is not unique.

Proof. The nonuniqueness of the representation will be evident from the proof of its existence which follows. For chains $c_{p}^{R}=0 \in C_{p}^{R}$, the result is trivial, so assume that $c_{p}^{R} \neq 0$. Then $c_{p}^{R}$ has a unique representation of the form

(i) $c_{p}^{R}=\sum_{j=1}^{n} m_{j}\left(v_{0 j}, \cdots, v_{p j}, T_{j}\right)^{R}$,

where the $m_{j}$ are nonzero integers and the $p$-cells $\left(v_{0 j_{1}}, \cdots, v_{p j_{1}}, T_{j_{1}}\right)^{R}$ and $\left(v_{0 j_{2}}, \cdots, v_{p j_{2}}, T_{j_{2}}\right)^{R}$ are distinct for $1 \leq j_{1} \leq j_{2} \leq n$. The proof is made by an induction on $n$. If $n=1$, then $c_{p}^{R}=m_{1}\left(v_{01}, \cdots, v_{p 1}, T_{1}\right)^{R}$. If, for some inte- 
gers $i, k$ such that $0 \leq i<k \leq p$, one finds $v_{i_{1}}=v_{k 1}$, then the fact that $c_{p}^{R}$ has a representation of the prescribed form follows from the lemma in $\$ 2.6$. On the other hand, if all the $v_{01}, \cdots, v_{p_{1}}$ are distinct, then $c_{p}^{R}$ is an elementary $n$-chain (see $\$ 2.7$ ). Thus the lemma is established in case $n=1$. Suppose that the lemma is true for all chains $c_{p}^{R}$ having a representation of the form (i) with at most $n=N-1$ terms $(N>1)$. For chains $c_{p}^{R}$ whose representations (i) have $N$ terms it is convenient to consider several cases.

Case 1. Assume there is some term in the representation (i) of $c_{p}^{R}$ - without loss of generality one may assume it to be the first - for which there are integers $i, k$ such that $0 \leq i<k \leq p$ and $v_{i 1}=v_{k 1}$. By the lemma in $\$ 2.6$ there are elements $t_{p 1}^{R}$ in $T_{p}^{R}$ and $d_{p 1}^{R}$ in $D_{p}^{R}$ such that

$$
m_{1}\left(v_{01}, \cdots, v_{p 1}, T_{1}\right)^{R}=t_{p 1}^{R}+d_{p_{1}}^{R} .
$$

By assumption there are elements $t_{p 2}^{R}$ in $T_{p}^{R}, d_{p 2}^{R}$ in $D_{p}^{R}$, and $n_{p}^{R}$ in $N_{p}^{R}$ such that

$$
\sum_{j=2}^{N} m_{j}\left(v_{0 j}, \cdots, v_{p j}, T_{j}\right)^{R}=t_{p 2}^{R}+d_{p 2}^{R}+n_{p}^{R}
$$

Thus

$$
c_{p}^{R}=\left(t_{p 1}^{R}+t_{p 2}^{R}\right)+\left(d_{p 1}^{R}+d_{p 2}^{R}\right)+n_{p}^{R},
$$

and since $T_{p}^{R}$ and $D_{p}^{R}$ are subgroups of $C_{p}^{R}$, the existence of a representation of the prescribed form for $c_{p}^{R}$ follows in Case 1 .

Case 2. Assume that for each $j(1 \leq j \leq N)$ the $v_{0 j}, \cdots, v_{p j}$ are distinct. By rearranging terms one may obtain from (i) a representation of the form

(ii) $c_{p}^{R}=\sum_{s=1}^{m} \sum_{r=1}^{n_{s}} m_{r s}\left(v_{0 s}, \cdots, v_{p s}, T_{r s}\right)^{R}, \sum_{s=1}^{m} n_{s}=N$,

satisfying these conditions: none of the $m_{r s}$ is zero; for the same $s(1 \leq s \leq m)$, $1 \leq r_{1}<r_{2} \leq n_{s}$, the mappings $T_{r_{1} s}$ and $T_{r_{2} s}$ are not identical on $\mid v_{0 s}$, $\cdots, v_{p s} \mid$; for $1 \leq s_{1}<s_{2} \leq m$, the $p$-cells $\left(v_{0 s_{1}}, \ldots, v_{p s_{1}}\right)$ and $\left(v_{0 s_{2}}\right.$, $\left.\cdots, v_{p s_{2}}\right)$ are distinct in $K($ see $\$ 1.1)$. Now for each $s(1 \leq s \leq m)$ clearly each of the chains

$$
c_{p s}^{R}=\sum_{j=1}^{n_{s}} m_{r s}\left(v_{0 s}, \cdots, v_{p s}, T_{r s}\right)^{R}
$$


is an elementary $n$-chain in $R$ (see $\$ 2.7$ ). The proof is carried forth by an inductive reasoning on $m$. If $m=1$ then $c_{p}^{R}$ is an elementary $n$-chain in $R$, and the representation (ii) already has the prescribed form. So assume that $c_{p}^{R}$, whose representation (i) has at most $N$ terms, has a representation of the prescribed form whenever its representation (ii) has at most $m=M-1$ terms $(M>1)$. Suppose now that $C_{p}^{R}$ is a chain whose representation (i) has $N$ terms while its representation (ii) has $M$ terms

$$
\sum_{s=1}^{M} n_{s}=N
$$

Subcase 2.1. Assume that for $1 \leq s_{1}<s_{2} \leq M$ the point sets $v_{0 s_{1}}, \cdots, v_{p s_{1}}$ and $v_{0 s_{2}}, \ldots, v_{p s_{2}}$ are distinct. From $\S 2.9$ it is clear that $c_{p}^{R}$ is itself an element in $N_{p}^{R}$ and representation (ii) has the prescribed form.

Subcase 2.2. Assume that there are distinct integers $s$ - with no loss of generality one may assume these to be $s=1$ and $s=2-$ such that the sets $v_{01}, \cdots, v_{p_{1}}$ and $v_{02}, \cdots, v_{p_{2}}$ are the same. It follows that the sequence $v_{02}, \cdots, v_{p_{2}}$ is obtainable from $v_{01}, \cdots, v_{p 1}$ by a positive number $l$ of transpositions. Hence by the lemma in $\$ 2.5$ there exists for each $r$ in $1 \leq r \leq n_{1}$ an element $t_{p r}^{R}$ in $T_{p}^{R}$ such that

$$
\left(v_{01}, \cdots, v_{p 1}, T_{r 1}\right)^{R}=(-1)^{l}\left(v_{02}, \cdots, v_{p 2}, T_{r_{1}}\right)^{R}+t_{p r}^{R} \quad\left(1 \leq r \leq n_{1}\right) .
$$

Since $T_{p}^{R}$ is a subgroup of $C_{p}^{R}$, the chain

$$
\sum_{r=1}^{n_{1}} m_{r_{1}} t_{p r}^{R}
$$

is an element $t_{p^{*}}^{R}$ in $T_{p}^{R}$. Consequently,

$$
\begin{aligned}
c_{p}^{R}=t_{p^{*}}^{R} & +\left[\sum_{r=1}^{n_{1}}(-1)^{l} m_{r 1}\left(v_{02}, \ldots, v_{p 2}, T_{r 1}\right)^{R}\right. \\
& \left.+\sum_{s=2}^{M} \sum_{r=1}^{n_{s}} m_{r s}\left(v_{0 s}, \ldots, v_{p s}, T_{r s}\right)^{R}\right] .
\end{aligned}
$$

Clearly the terms in square brackets may be rearranged into the form (ii) with an integer $m \leq M-1$, and the ir representation in form (i) has an integer $n \leq N$. By the inductive assumption there are elements $t_{p \#}^{R}$ in $T_{p}^{R}, d_{p}^{R}$, in $D_{p}^{R}$ and $\overline{n_{p}^{R}}$ in 
$N_{p}^{R}$ such that $c_{p}^{R}=\left(t_{p}^{R}+t_{p \#}^{R}\right)+d_{p}^{R}+n_{p}^{R}$, and the existence of a representation of the prescribed form for $c_{p}^{R}$ now follows in Case 2. Indeed, it is obvious in this case that $d_{p}^{R}=0 \in C_{p}^{R}$. So the lemma is completely established.

2.13. LEMmA. If $c_{p}^{R}$ is any chain in $C_{p}^{R}$ for which $\beta_{p}^{R} c_{p}^{R}=0$, then

$$
\beta_{p+{ }_{1}}^{R} \rho_{* p}^{R} c_{p}^{R}=0
$$

The proof follows at once from the lemmas in $\$ \$ 2.3,2.4,2.11,2.12$.

\section{RESULTS}

3.1. In [3, \$4.1] (see also [4, §8]) Radó has established a lemma from which one derives the following statement by replacing the barycentric homotopy operator $\rho_{p}^{R}$ by the modified barycentric homotopy operator $\rho_{* p}^{R}($ see $\S \S 1.2,2.1)$.

LEмMA. Let $\left\{G_{p}\right\}$ be an identifier for $R$ (see $\$ 0.3$ ) such that the following conditions hold:

(i) $c_{p}^{R} \in G_{p}$ implies that $\beta_{p}^{R} c_{p}^{R}=0$;

(ii) $c_{p}^{R} \in G_{p}$ implies that $\rho_{* p}^{R} c_{p}^{R} \in G_{p+1}$.

Then $\left\{G_{p}\right\}$ is unessential.

3.2. For each integer $p$ let $N\left(\beta_{p}^{R}\right)$ be the nucleus of the homomorphism $\beta_{p}^{R}: C_{p}^{R} \rightarrow C_{p}^{R}$ (see $\$ 2.1$ ). Since $\beta_{p}^{R}$ is a chain mapping (see $\$ 2.2$ ) it is clear that the nuclei $N\left(\beta_{p}^{R}\right)$ constitute an identifier for $R$ (see $\$ 0.3$ ). Now in view of the lemma in $\$ 2.13$, conditions (i) and (ii) of the lemma above are clearly fulfilled for the identifier $\left\{N\left(\beta_{p}^{R}\right)\right\}$, and furthermore, this choice of an identifier yields the maximum amount of information that may be obtained from that lemma. Thus the $\left\{N\left(\beta_{p}^{R}\right)\right\}$ constitute an unessential identifier for $R$, and one of the main results is now established (see $\$ 0.4$ ). It is summarized in the following statement.

THE OREM. The system of nuclei $N\left(\beta_{p}^{R}\right)$ of the barycentric homomorphisms $\beta_{p}^{R}: C_{p}^{R} \longrightarrow C_{p}^{R}$ constitutes an unessential identifier for $R$.

3.3. In order to compare this result with those in Rado [3; 4], first observe that it follows from the lemmas in $\$ \S 2.3,2.4$ that

$$
N\left(\beta_{p}^{R}\right) \supset T_{p}^{R}+D_{p}^{R} \quad(-\infty<p<+\infty) .
$$

Moreover, since $C_{p}^{R}$ is a free group, it is clear that the division hull of $N\left(\beta_{p}^{R}\right)$ 
must be identical with the group $N\left(\beta_{p}^{R}\right)$. Thus the group $N\left(\beta_{p}^{R}\right)$ also contains the the division hull of the group $T_{p}^{R}+D_{p}^{R}$ for all integers $p$. An example is now given to show that the group $N\left(\beta_{p}^{R}\right)$ generally contains more.

3.4. Denote by $d_{0}, d_{1}, d$ the points $(1,0,0, \ldots),(0,1,0,0, \ldots),(1 / 2$, $1 / 2,0,0, \ldots)$ respectively, let $X$ be Euclidean $x$-space, and define transformations by the following relations:

$$
\begin{aligned}
& T_{1}: x=v_{0}-1 / 2 \quad\left(v \in\left|d_{0}, d_{1}\right|\right) ; \\
& T_{2}: x= \begin{cases}0 & \left(v \in\left|d_{0}, d\right|\right) ; \\
v_{0}-1 / 2 & \left(v \in\left|d, d_{1}\right|\right) ;\end{cases} \\
& T_{3}: x= \begin{cases}v_{0}-1 / 2 & \left(v \in\left|d_{0}, d\right|\right) ; \\
0 & \left(v \in\left|d, d_{1}\right|\right) ;\end{cases} \\
& T_{4}: x=0 \quad\left(v \in\left|d_{0}, d_{1}\right|\right) \text {. }
\end{aligned}
$$

Clearly

$$
c_{1}^{R}=\left(d_{0}, d_{1}, T_{1}\right)^{R}-\left(d_{0}, d_{1}, T_{2}\right)^{R}-\left(d_{0}, d_{1}, T_{3}\right)^{R}+\left(d_{0}, d_{1}, T_{4}\right)^{R}
$$

belongs to $C_{1}^{R}$ and $\beta_{1}^{R} c_{1}^{R}=0$. Moreover, $c_{1}^{R}$ is an elementary $n$-chain (see §2.7). An elementary reasoning shows that it cannot belong to the division hull for the group $T_{1}^{R}+D_{1}^{R}$.

3.5. In order to describe the largest unessential identifier for $R$ obtained by Radó, a further definition is needed. For integers $p \geq 0$, let $\left(v_{0}, \cdots, v_{p}, T\right)^{R}$ bé any $p$-cell in $R$ (see $\$ 0.1$ ). Let $w_{0}, \cdots, w_{p}$ be any set sequence of $p+1$ linearly independent points in $E_{\infty}$. Then there is a linear mapping

$$
\alpha:\left|w_{0}, \cdots, w_{p}\right| \rightarrow \mid v_{0}, \cdots, v_{p} .
$$

such that $\alpha\left(w_{i}\right)=v_{i}$ for $0 \leq i \leq p$. The $p$-chain

$$
c_{p}^{R}=\left(v_{0}, \cdots, v_{p}, T\right)^{R}-\left(w_{0}, \cdots, w_{p}, T \alpha\right)^{R}
$$

is termed an elementary a-chain in $R$ (see [3, $\$ 3.4]$ ), and the subgroup of $C_{p}^{R}$ generated by the elementary $a$-chains is denoted by $A_{p}^{R}$. For $p<0, A_{p}^{R}$ consists of the zero element alone. In [3, \$3.4] Radó has a simple characterization for the group $A_{p}^{R}$ which he uses to define the group in $[4, \& 7]$.

3.6. For each integer $p$, put $\Gamma_{p}^{R}=A_{p}^{R}+D_{p}^{R}+T_{p}^{R}$ (see $\$ \$ 2.3,2.4,3.5$ ), and let $\hat{\Gamma}_{p}^{R}$ denote the division hull of $\Gamma_{p}^{R}$. Then Radó shows that $\left\{\hat{\Gamma}_{p}^{R}\right\}$ is an 
unessential identifier in $R$ (see $[3, \S 4.7]$ or $[4, \S 9]$ ), and this is his best result. If one sets $\Delta_{p}^{R}=A_{p}^{R}+N\left(\beta_{p}^{R}\right)$ (see $\left.\$ 3.2\right)$ and lets $\hat{\Delta}_{p}^{R}$ denote the division hull of $\Delta_{p}^{R}$, then clearly $\Delta_{p}^{R} \supset \Gamma_{p}^{R}$, and hence $\hat{\Delta}_{p}^{R} \supset \hat{\Gamma}_{p}^{R}$. If one modifies the reasoning of Radó in $[3, \S 4]$ by replacing the barycentric homotopy operator $\rho_{p}^{R}$ by the modified barycentric homotopy operator $\rho_{* p}^{R}$ (see $\$ 2.1$ ), one finds that $\hat{\Delta}_{p}^{R}$ is an unessential identifier for $R$. Thus one obtains the following result.

THEOREM. If $\hat{\Delta}_{p}^{R}$ is the division hull of the group $A_{p}^{R}+N\left(\beta_{p}^{R}\right)$ then the system $\left\{\hat{\Delta}_{p}^{R}\right\}$ is an unessential identifier for $R$.

\section{REFERENCES}

1. S. Eilenberg and N. E. Steenrod, Foundations of Algebraic Topology (unpublished).

2. T. Radó, On identifications in singular homology the ory, Rivista Mat. Univ. Parma, 2 (1951), 3-18.

3. 265-290.

4. - On the foundations of singular homology theory, to appear in Mem. Accad. Sci. Ist. Bologna, Cl. Sci. Fis.

Ohio State University 



\section{EDITORS}

HERBERT BUSEMANN

University of Southern California

Los Angeles 7, California
R. M. ROBINSON

University of California

Berkeley 4, California

E. F. BECKENBACH, Managing Editor

University of California

Los Angeles 24, California

\section{ASSOCIATE EDITORS}

\author{
R. P. DILWORTH \\ HERBERT FEDERER \\ MARSHALL HALL
}

P. R. HALMOS

HEINZ HOPF

R. D. JAMES

\author{
BØRGE JESSEN \\ PAUL LÉVY \\ GEORGE PÓLYA
}

J. J. STOKER
E. G. STRAUS

KÖSAKU YOSIDA

\section{SPONSORS}

UNIVERSITY OF BRITISH COLUMBIA

CALIFORNLA INSTITUTE OF TECHNOLOGY

UNIVERSITY OF CALIFORNIA, BERKELLEY

UNIVERSITY OF CALIFORNIA, DAVIS

UNIVERSITY OF CALIFORNIA, LOS ANGELES

UNIVERSTTY OF CALIFORNIA, SANTA BARBARA

OREGON STATE COLLEGE

UNIVERSITY OF OREGON
UNIVERSETY OF SOUTHERN CALIFORNIA STANFORD UNIVERSITY

WASHINGTON STATE COLLEGE UNIVERSTTY OF WASHINGTON

AMERICAN MATHEMATICAL SOCIETY NATIONAL BUREAU OF STANDARDS, INSTITUTE FOR NUMERICAL ANALYSIS

Vari-Type Composition by

Elaine Barth

Delores Gilbertson

With the cooperation of

E. F. Beckenbach

E. G. Straus

Printed in the United States of America by

Edwards Brothers, Inc., Ann Arbor, Michigan

UNIVERSITY OF CALIFORNIA PRESS * BERKELEY AND LOS ANGELES

COPYRIGHT 1952 BY PACIFIC JOURNAL OF MATHEMATICS 


\section{Pacific Journal of Mathematics}

\section{Vol. 2, No. $1 \quad$ January, 1952}

Tom M. (Mike) Apostol, Theorems on generalized Dedekind sums ........ 1

Tom M. (Mike) Apostol, Addendum to 'On the Lerch zeta function' ........ 10

Richard Arens, Extension of functions on fully normal spaces ........... 11

John E. Maxfield, A short proof of Pillai's theorem on normal numbers.... 23

Charles B. Morrey, Quasi-convexity and the lower semicontinuity of multiple integrals................................ 25

P. M. Pu, Some inequalities in certain nonorientable Riemannian manifolds....................................... 55

Paul V. Reichelderfer, On the barycentric homomorphism in a singular

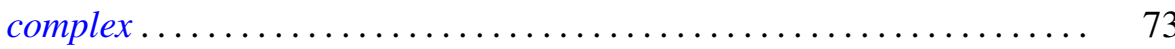

A. H. Stone, Incidence relations in multicoherent spaces. III ........... 99 